\title{
Visibility Graphs and Oriented Matroids (Extended Abstract)
}

\author{
James Abello ${ }^{\star 1}$ and Krishna Kumar ${ }^{2}$ \\ 1 Department of Computer Science, Texas A\&M University, College Station, TX \\ 77843, USA \\ 2 Department of Mathematics and Computer Science \\ Colby College, Waterville, ME 04901, USA.
}

\begin{abstract}
This paper describes a new set of necessary conditions for a given graph to be the visibility graph of a simple polygon. For every graph satisfying these conditions we show that a uniform rank 3 oriented matroid can be constructed in polynomial time, which if affinely co- ordinatizable would yield a simple polygon whose visibility graph is isomorphic to the given graph. This will in turn offer the first characterization of this class of graphs.
\end{abstract}

\section{Introduction}

Visibility graphs are fundamental structures in computational geometry. They find applications in areas such as graphics $[13,21]$ and robotics [16], yet very little is known about their combinatorial structure. This paper addresses the question of characterizing internal visibility graphs of simple plane polygons, henceforth simply called visibility graphs. Two vertices of a simple polygon $P$, are called visible, if the open line segment between them is either a boundary edge of $P$, or is completely contained in the interior of the polygon. Note that in this setting, two vertices are considered to be invisible if the open line segment between them passes through a third vertex of the polygon. The visibility graph of a polygon is the graph whose vertices correspond to the vertices of the polygon and edges correspond to visible pairs of vertices in the polygon. From the computational standpoint, the complexity of the recognition problem for visibility graphs is only known to be in PSPACE [9]. It is not known to be in $N P$ nor it is known to be $N P$-complete.

Visibility graphs do not lie in any of the well known classes of graphs such as planar graphs, perfect graphs etc. $[9,11]$. The first set of necessary conditions for a graph to be a visibility graph were obtained by Ghosh [11]. However, it was

\footnotetext{
* Work partially supported by NSF grants No: DCR-8603722 and DCR-8896281
} 
shown by Everett [9] that these conditions were not sufficient. Further necessary conditions were developed by Coullard and Lubiw [8], but they also showed that they are not sufficient. Abello, Hua and Pisupati [2] have strengthened these results by showing that the proposed conditions are not sufficient, even for triconnected graphs, and in the case of the conditions of [8], even for planar graphs. O'Rourke [19] has an excellent review of current status of this research. In this paper, we develop stronger necessary conditions for a graph to be a visibility graph.

In order to show that a given set of conditions on a graph, are sufficient for the graph to be a visibility graph, one must demonstrate that every graph satisfying the conditions can be realized as the visibility graph of a simple polygon in the plane. However, this reconstruction problem appears to be quite difficult in the general case. In this paper, we solve a combinatorial version of the reconstruction problem for general visibility graphs. We prove new necessary conditions for visibility graphs and show that these conditions are sufficient to construct a uniform oriented matroid of rank 3 corresponding to each graph in this class. These oriented matroids are combinatorial representations of simple polygons realizing the graphs, in the sense that any affine realization of the oriented matroids yields a simple polygon whose visibility graph is isomorphic to the given graph. It would be sufficient to show that each of these oriented matroids is affinely realizable, in order to obtain a characterization of visibility graphs of simple polygons. The main results of the paper are summarized below.

1. A class of graphs called Quasi-Persistent graphs is defined and it is shown that visibility graphs are properly contained in this class.

2. Several new necessary conditions are proven for a given Quasi-Persistent graph to be a visibility graph. These conditions strengthen Ghosh's necessary conditions for visibility graphs.

3. For each Quasi-Persistent graph satisfying these necessary conditions, a uniform oriented matroid of rank 3 is constructed (in polynomial time) such that any affine realization of the oriented matroid yields a simple polygon whose visibility graph is isomorphic to the given graph.

Because of space restrictions, we just give the main ideas involved in the proofs. The details may be found in [4] and [14].

\section{Definitions}

It is clear that every visibility graph is Hamiltonian and we therefore restrict our attention to Hamiltonian graphs. We further assume that the graphs considered are undirected, loopless and do not have multiple edges.

Let $G=(V, E)$ be a Hamiltonian graph with a prescribed Hamiltonian cycle $H$. The vertices of $G$ are labelled along $H$ from 0 to $n-1$. The vertex labelled $i$ is denoted $v_{i}, v_{i-1}$ and $v_{i+1}$ respectively, denote the predecessor and successor of $v_{i}$ on $H$. All subscript arithmetic is modulo $n$. It will be convenient to think 
of $G$ as being embedded in the plane so that $H$ forms a simple closed curve. In this setting, a traversal of $H$ from $v_{i}$ to $v_{j}$ in the order $v_{i}, v_{i+1}, \ldots, v_{j-1}, v_{j}$ may be thought of as a counterclockwise traversal of $H$, and the traversal that goes from $v_{i}$ to $v_{j}$ in the order $v_{i}, v_{i-1}, \ldots, v_{j+1}, v_{j}$ will correspond to clockwise traversals. In this paper, unless specified otherwise, traversals of $H$ are implicitly assumed to be in counterclockwise order.

For any two vertices $v_{i}$ and $v_{j}$, the ordered set $\left\{v_{i}, v_{i+1}, \ldots, v_{j-1}, v_{j}\right\}$ of vertices encountered in traversing $H$ from $v_{i}$ to $v_{j}$, is called the chain from $v_{2}$ to $v_{j}$ and is denoted chain $\left[v_{i}, v_{j}\right]$. This set of vertices constitutes a simple path in $G$. The chain from $v_{i+1}$ to $v_{j-1}$ is denoted chain $\left(v_{i}, v_{j}\right)$. We also use chain $\left[v_{i}, v_{j}\right)$ and $\operatorname{chain}\left(v_{i}, v_{j}\right]$ in the obvious manner. We emphasize that $\operatorname{chain}\left(v_{i}, v_{k}\right)$ and $\operatorname{chain}\left(v_{k}, v_{i}\right)$ are always disjoint sets. We say that $v_{\imath}<v_{j}<v_{k}$ if $v_{j}$ lies on $\operatorname{chain}\left(v_{i}, v_{k}\right)$.

Two vertices $v_{i}$ and $v_{j}$ of $G$ are said to be invisible if $v_{i} v_{j} \in \bar{E}$. For an invisible pair $v_{i}, v_{k}$, a vertex $v_{j}$ is called an inner blocking vertex [11], relative to $H$, if $v_{j}$ lies on $\operatorname{chain}\left(v_{i}, v_{k}\right)$ and $v_{x} v_{y} \in \bar{E}$ for all $v_{x}$ on $\operatorname{chain}\left[v_{i}, v_{j}\right)$ and $v_{y}$ on chain $\left(v_{j}, v_{k}\right]$. Similarly, a vertex $v_{j}$ is called an outer blocking vertex relative to $H$ for the invisible pair $v_{\imath} v_{k}$ if $v_{j}$ lies on $\operatorname{chain}\left(v_{k}, v_{i}\right)$ and $v_{x} v_{y} \in \bar{E}$ for all $v_{x}$ on $\operatorname{chain}\left(v_{j}, v_{\imath}\right]$ and $v_{y}$ on chain $\left[v_{k}, v_{j}\right)$. In general, $v_{j}$ is called a blocking vertex for the invisible pair $v_{i} v_{k}$ if it is either an inner or an outer blocking vertex for this pair.

A simple path $P=u_{0} u_{1} \ldots u_{r}$ is called an ordered path relative to $H$, if the vertices in $P$ are encountered in the order $u_{0}, u_{1}, \ldots, u_{r}$ when $H$ is traversed from $u_{0}$. Similarly, a simple cycle $C=u_{0} u_{1} \ldots u_{r} u_{0}$ is called an ordered cycle relative to $H$ if the vertices in $C$ are encountered in the order $u_{0}, u_{1}, \ldots u_{r}, u_{0}$ (or its reverse) when $H$ is traversed from $u_{0}$. Two pairs $v_{2} v_{3}$ and $v_{k} v_{l}$, are said to be separable [11] with respect to a vertex $v_{p}$ if both $v_{2}$ and $v_{j}$ are encountered before $v_{k}$ and $v_{l}$ (or vice versa), when $H$ is traversed from $v_{p}$. In this case, we say that $v_{\imath} v_{3}$ and $v_{k} v_{l}$ are $v_{p}$-separable; otherwise we say that they are $v_{p}$-inseparable. Note that two pairs $v_{\imath} v_{j}$ and $v_{k} v_{l}$ are separable with respect to $v_{p}$ when the two pairs do not interlace on the boundary, ( i.e $v_{\imath}<v_{\jmath}<v_{k}<v_{l}$ ) and $v_{p}$ lies on $\operatorname{chain}\left(v_{\jmath}, v_{k}\right)$ or on $\left.\operatorname{chain}\left(v_{k}, v_{\imath}\right)\right)$.

We now introduce a new class of graphs called Quasi-Persistent graphs, and show that the visibility graphs of all simple polygons are contained in this class. This class is a natural generalization of persistent graphs, a class originally introduced by Abello and Egecioglu [1].A graph $G$ with Hamiltonian cycle $H$, is said to be Quasi-Persistent (or q-persistent) relative to $H$, if for every triple of vertices $v_{i}<v_{p}<v_{q}$, such that $v_{i} v_{p}$ and $v_{i} v_{q} \in E$, and $v_{i} v_{j} \in \bar{E}$, for all $v_{j}$ in chain $\left(v_{p}, v_{q}\right)$, the following conditions hold

1. $v_{p}$ is adjacent to $v_{q}$.

2. For every $v_{j}$ in chain $\left(v_{p}, v_{q}\right)$, at least one of the vertices $v_{p}$ or $v_{q}$ is a blocking vertex for $v_{\imath} v_{j}$.

The graph in figure 1 is a q-persistent graph. For a pair $v_{i} v_{j}$ of non-consecutive vertices, let $v_{p}$ be the first vertex adjacent to $v_{\imath}$, that is encountered on a clockwise traversal of $H$, starting from $v_{\jmath-1} . v_{p}$ is called the first neighbour of $v_{\imath}$ 


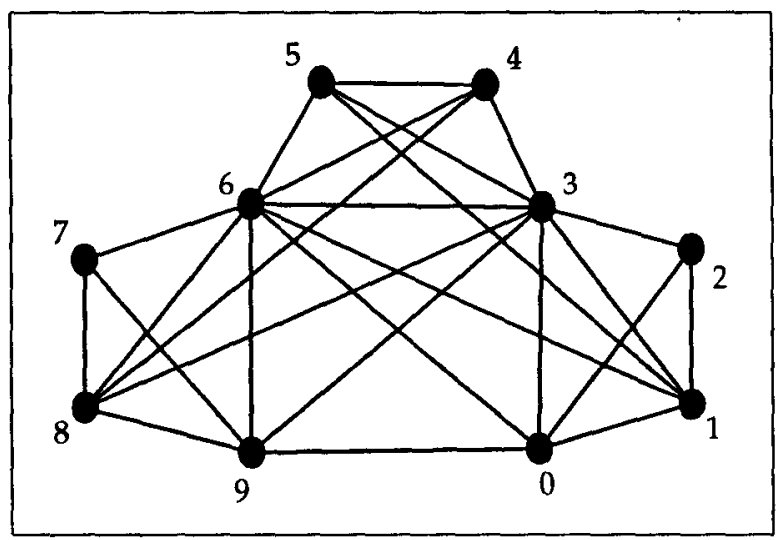

Fig. 1. A q-persistent graph.

before $v_{j}$ and is denoted as $\operatorname{pn}\left(v_{i} v_{j}\right)$. Similarly, the first vertex $v_{q}$ adjacent to $v_{\imath}$, encountered on a counter-clockwise traversal of $H$ from $v_{j+1}$, is called the first neighbour of $v_{z}$ after $v_{j}$ and is denoted $\operatorname{sn}\left(v_{z} v_{j}\right)$. Since $G$ is Hamiltonian, $\operatorname{pn}\left(v_{i} v_{j}\right)$ and $\operatorname{sn}\left(v_{i} v_{j}\right)$ exist for every invisible pair $v_{i} v_{j}$, and they are distinct. Also, note that the definition is not symmetric, i.e, it is not necessary that $\operatorname{pn}\left(v_{i} v_{j}\right)$ and $\operatorname{sn}\left(v_{i} v_{j}\right)$ be the same as $\operatorname{pn}\left(v_{j} v_{i}\right)$ and $\operatorname{sn}\left(v_{j} v_{i}\right)$ respectively. The q-persistence conditions imply that for any invisible pair $v_{i} v_{j}$, the vertices $\operatorname{pn}\left(v_{i} v_{j}\right)$ and $\operatorname{sn}\left(v_{i} v_{j}\right)$ are adjacent in $G$, and at least one of them is a blocking vertex for $v_{i} v_{j}$.

Ghosh [11] gave the first set of necessary conditions for a given graph to be a visibility graph. These conditions which we will henceforth call Ghosh's conditions are summarized below.

Proposition 1. [Ghosh]If a graph $G$ is the visibulity graph of a simple polygon then

1. G has Hamiltonian cycle $H$.

2. Every ordered cycle relative to $H$ of length $\geq 4$ has a chord.

3. Every invisible pair in $G$ has a blocking vertex relative to $H$.

4. If two invisible pairs are separable with respect to a vertex $v_{p}$, then $v_{p}$ cannot be the only blocking vertex for both the invisible pairs.

Our q-persistent graphs satisfy the first and third conditions of proposition 1 by definition. In fact, the second q-persistence condition(ordered chordality) appears, at first glance, to be much stronger than Ghosh's third condition. However, it can be shown that the class of q-persistent graphs is equivalent to the class of graphs that satisfies the first three of Ghosh's conditions. We summarize this as theorem 2 below.

Theorem 2. A graph $G$ with Hamiltonian cycle $H$ is q-persistent relative to $H$ if and only if every ordered cycle of length $\geq 4$ has a chord and every invisible pair has a blocking vertex (relative to $H$ ). 
Thus, q-persistent graphs are not a fundamentally new class of graphs. The main advantage of the above formulation is that the simpler structure of the definition makes it easier to analyze and prove properties of the resulting class. It is interesting to note the relationship between the two q-persistence conditions and Ghoshs conditions 2 and 3. The first q-persistence condition is a "weaker" version of ordered chordality, in the sense that the graphs that are Hamiltonian and ordered chordal are properly contained in the class of (Hamiltonian) graphs satisfying the first q-persistence condition. On the other hand the second q-persistence condition is a stronger version of Ghosh's condition 3 since Hamiltonian graphs that satisfy the second q-persistence condition are properly contained in the class of Hamiltonian graphs satisfying Ghosh's condition 3 . However, when both pairs of conditions are considered together, the classes become equivalent!

Since visibility graphs satisfy all four of Ghosh's conditions, it is evident from theorem 2 that visibility graphs are properly contained in the class of qpersistent graphs. The following section develops additional necessary conditions for a q-persistent graph to be a visibility graph and shows that these conditions are strictly stronger than Ghosh's conditions.

\section{New Necessary Conditions for Visibility Graphs}

We assume throughout this section, that $P$ is a simple polygon in the Euclidean plane and that a q-persistent graph $G$ is its visibility graph. Arbitrary points of the plane are denoted as $p_{x}, p_{y}$ etc. For two points $p_{x}$ and $p_{y}$, the ray from $p_{x}$ in the direction of $p_{y}$ will be denoted $\boldsymbol{r}_{x y}$. For a vertex $v_{z}$ of $G$ the corresponding vertex of $P$ is denoted $v_{\imath}^{*}$. We will also use $\boldsymbol{r}_{\imath \jmath}$ to denote the ray from $v_{z}^{*}$ in the direction of $v_{\jmath}^{*}$. A polygon $P$ whose visibility graph is $G$, is called a realization of the graph $G$. A given q-persistent graph that is a visibility graph, can have many different realizations.

Suppose $W=w_{0}, \ldots, w_{k}$ is the sequence of neighbors of a vertex $v_{\imath}$ in $G$, obtained in traversing $H$, with $w_{0}=v_{t+1}$ and $w_{k}=v_{t-1}$. In a polygon $P$ realizing $G, \angle w_{k}^{*} v_{\imath}^{*} w_{\jmath-1}^{*}<\angle w_{k}^{*} v_{i}^{*} w_{\jmath}^{*}$ for $1 \leq j \leq k-1$ ( see [9], pg. 18 for a proof of this fact). The second q-persistence condition can now be interpreted geometrically. let $v_{\imath}<v_{p}<v_{q}$ be a triple of vertices in $G$ such that $v_{\imath} v_{p}, v_{i} v_{q} \in E$ and $v_{\imath} v_{\jmath} \in \bar{E}$ for all $v_{j}$ on chain $\left(v_{p}, v_{q}\right)$. For the corresponding triple of points $v_{\imath}^{*}, v_{p}^{*}$, and $v_{q}^{*}$ in a realization $P$ of $G$, there exists a unique segment $v_{k}^{*} v_{k+1}^{*}$ on the boundary of $P$, such that, $v_{k}$ and $v_{k+1}$ lie on chain $\left[v_{p}, v_{q}\right]$, and for any ray $\boldsymbol{r}_{i x}$ such that $\angle v_{i-1}^{*} v_{i}^{*} v_{p}^{*}<\angle v_{i-1}^{*} v_{i}^{*} p_{x}<\angle v_{i-1}^{*} v_{i}^{*} v_{q}^{*}$, the first segment on the boundary of $P$ that is intersected by ray $\boldsymbol{r}_{i x}$ is $v_{k}^{*} v_{k+1}^{*}$. The fact to be emphasized here is that the segment so obtained does not depend on the specific ray $\boldsymbol{r}_{2 x}$, but only on the triple of points involved (see figure 2).

For any vertex $v_{j}$ on chain $\left(v_{p}, v_{k}\right]$, the vertex $v_{p}$ is a blocking vertex ( in $G$ ) for $v_{i} v_{j}$ and for any vertex $v_{j}$ on chain $\left[v_{k+1}, v_{q}\right)$, the vertex $v_{q}$ is a blocking vertex for $v_{\imath} v_{\jmath}$. The edge $v_{k}^{*} v_{k+1}^{*}$ is called the split segment for 


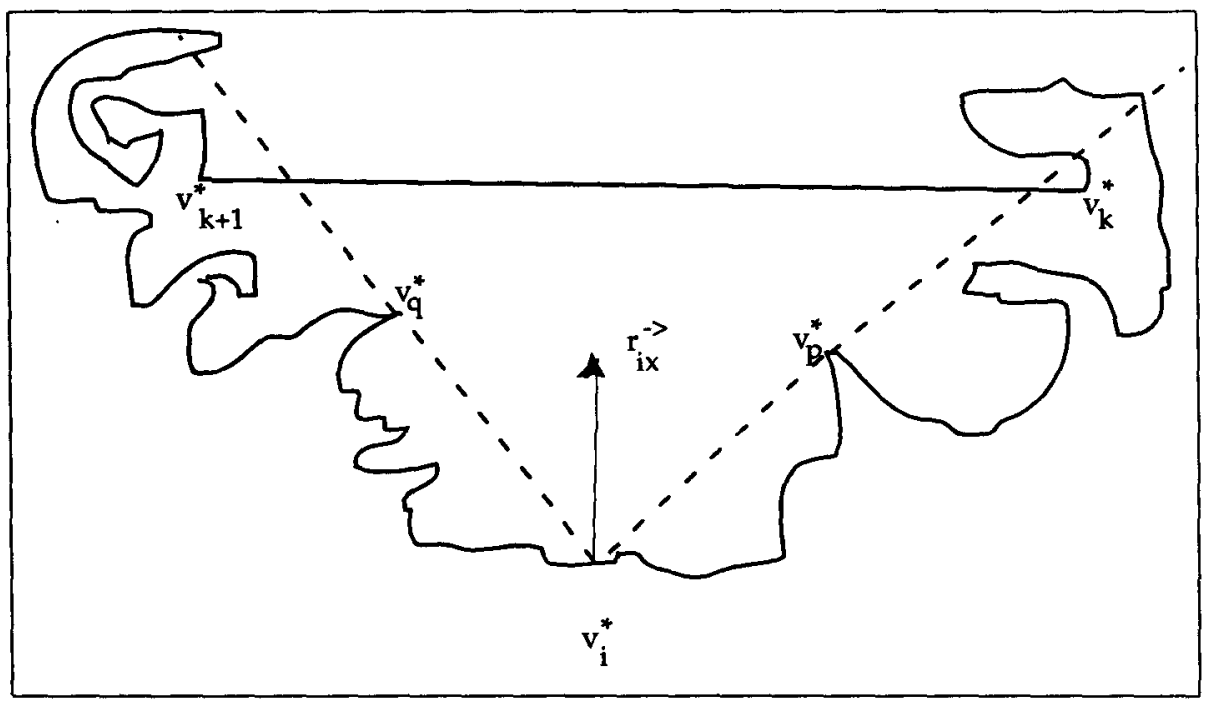

Fig. 2. Geometric interpretation of q-persistence

the triple of points $v_{i}^{*} v_{p}^{*} v_{q}^{*}$. The corresponding edge in the graph is called the split edge. Intuitively, the split edge determines, which one of the points $v_{p}^{*}$ and $v_{q}^{*}$ is involved in "physically" blocking a given pair $v_{i} v_{j}$ on $\operatorname{chain}\left(v_{p}, v_{q}\right)$ in a given polygon whose visibility graph is $G$. In general, the split edge is not determined by the visibility graph alone. Different polygons with the same underlying visibility graph may have different split edges for the same triple of vertices in $G$.

The q-persistence conditions stipulate that for any invisible pair $v_{\imath} v_{j} \in \bar{E}$ of a q-persistent graph $G$, at least one of the vertices $\operatorname{pn}\left(v_{i} v_{j}\right)$ or $\operatorname{sn}\left(v_{i} v_{j}\right)$ must be a blocking vertex for the invisible pair. However, according to the discussion above, in any fixed realization of $G$, at most one of these vertices "physically" blocks the corresponding invisible pair of points in the realization. This motivates the following definitions.

A vertex $v_{p}$ is called a primary blocking vertex for an invisible pair $v_{\imath} v_{j}$ if $v_{p}$ is a blocking vertex for $v_{i} v_{j}$ and $v_{i} v_{p} \in E$. By the definition of blocking vertices, the only possible choices for the primary blocking vertices for $v_{i} v_{j}$ are $\operatorname{pn}\left(v_{i} v_{j}\right)$ and $\operatorname{sn}\left(v_{i} v_{j}\right)$. Therefore, if either of the vertices $\operatorname{pn}\left(v_{i} v_{j}\right)$ or $\operatorname{sn}\left(v_{i} v_{j}\right)$ is a blocking vertex for the invisible pair $v_{i} v_{j}$ in a q-persistent graph $G$, then it is called a primary blocking vertex for $v_{\imath} v_{3}$. The q-persistence conditions imply that every invisible pair has at least one primary blocking vertex. Also, the primary blocking vertices of the pair $v_{i} v_{j}$ are not necessarily the same as those for $v_{j} v_{i}$.

A blocking vertex assignment ${ }^{3}$ for a q-persistent graph $G$, is a function

${ }^{3}$ Everett, in [9], also defines a similar notion, but the requirement that vertices in the image of the function be primary blocking vertices makes the definition given here strictly stronger than the one in [9] 
$\beta: \bar{E} \rightarrow V$ such that, for all $v_{i} v_{j} \in \bar{E}, \beta\left(v_{i} v_{j}\right)$ is a primary blocking vertex for $v_{i} v_{j}$. Any q-persistent graph has at least one blocking vertex assignment. If $G$ is a visibility graph, then every fixed realization, $P$ of a given q-persistent graph, determines a particular blocking vertex assignment for $G$ as follows. For a triple $v_{i}<v_{p}<v_{q}$ of vertices in $G$, such that $v_{i} v_{p}, v_{i} v_{q} \in E$ and $v_{i} v_{j} \in \bar{E}$ for all $v_{j}$ on chain $\left(v_{p}, v_{q}\right)$, let $v_{k}^{*} v_{k+1}^{*}$ be the split segment in $P$ for the triple $v_{i}^{*} v_{p}^{*} v_{q}^{*}$. We set $\beta\left(v_{i} v_{j}\right)=v_{p}$ for all $v_{j}$ on $\operatorname{chain}\left(v_{p}, v_{k}\right]$. For all $v_{j}$ on chain $\left[v_{k+1}, v_{q}\right)$ we set $\beta\left(v_{i} v_{j}\right)=v_{q}$. From the discussion in the last section, it follows that $\beta$ is a blocking vertex assignment for $G$. This blocking vertex assignment is called a canonical blocking vertex assignment for $G$ determined by the realization $P$.

We now consider the following problem: Given a q-persistent graph together with a blocking vertex assignment $\beta$, determine the conditions under which there exists a polygon $P$ whose visibility graph is $G$, and such that the canonical assignment on $G$ determined by $P$ is $\beta$. Such conditions will clearly yield a characterization of visibility graphs. It turns out that blocking vertex assignments on q-persistent graphs must satisfy four additional necessary conditions in order to be canonical assignments.

A blocking vertex assignment is said to be locally inseparable if any two invisible pairs $v_{i} v_{j}$ and $v_{k} v_{l}$ such that $\beta\left(v_{i} v_{j}\right)=\beta\left(v_{k} v_{l}\right)=v_{p}$ are $v_{p}$-inseparable ( see definition on page 3 ). The following is a necessary condition for a blocking vertex assignment to be a canonical blocking vertex assignment ${ }^{4}$.

Necessary Condition 1: If $\beta$ is a canonical blocking vertex assagnment for a $q$-persistent graph $G$, determined by a realization $P$, then $\beta$ is locally-inseparable.

In order to state the remainmng necessary conditions we need to introduce the following definition Given an invisible pair $v_{\imath} v_{k}$ in $G$, an occluding path generated by $\beta$, between $v_{2}$ and $v_{k}$, denoted $\operatorname{path}_{\beta}\left(v_{\imath}, v_{k}\right)$ is a path $v_{\imath} u_{0} \ldots u_{r} v_{k}$ in $G$, such that $u_{0}=\beta\left(v_{\imath} v_{k}\right), u_{\jmath} v_{k} \in \bar{E}$, and $u_{\jmath+1}=\beta\left(u_{j}, v_{k}\right)$ for $0 \leq j \leq r-1$. It is readıly seen that a given blockıng vertex assignment determines a unique occluding path between every invisible pair of vertices. It can also bee shown that this path is simple and that every internal vertex on this path is a blocking vertex for the invisible pair. For notational convenience, we identify $\operatorname{path}_{\beta}\left(v_{\imath}, v_{k}\right)$ with its underlying set of vertices.

When a graph $G$ is the visibility graph of a polygon $P$, the graph theoretical notion of occluding path corresponds to the geometric notion of shortest path under the geodesic metric. This fact is stated in the following proposition

Proposition. 3. Let $\beta$ be the canonical blocking vertex assignment for a visibulity graph $G$, determined by a fixed realization $P$. A vertex $v_{x}$ lies on path ${ }_{\beta}\left(v_{2}, v_{\jmath}\right)$ if and only if $v_{x}^{*}$ lues on the Euclidean shortest path in $P$ between $v_{\imath}^{*}$ and $v_{j}^{*}$.

${ }^{4}$ Everett [9] conjectures a similar result. However, since our definition of blocking vertex assignment is stricter, Necessary Condition 1 is stronger. 
The remaining necessary conditions arise as a result of this correspondence between occluding and Euclidean shortest paths. A blocking vertex assignment is called path-symmetric if for every invisible pair $v_{i} v_{k}$ such that $\operatorname{path}_{\beta}\left(v_{\imath}, v_{k}\right)=v_{i} u_{0} \ldots u_{r} v_{k}$, we have $\operatorname{path}_{\beta}\left(v_{k}, v_{i}\right)=v_{k} u_{r} \ldots u_{0} v_{\imath}$. We denote this as $\operatorname{path}_{\beta}\left(v_{k}, v_{i}\right)=\operatorname{path}_{\beta}^{R}\left(v_{i}, v_{k}\right)$ In other words, even though a blocking vertex assignment is not symmetric in the blocking vertices it assigns to invisible pairs $v_{i} v_{k}$ and $v_{k} v_{i}$, it must ensure the symmetry of the occluding paths generated under the assignment between every invisible pair of vertices. Since Euclidean shortest paths between two points inside a simple polygon are unique, it readily follows that canonical blocking vertex assignments must be path-symmetric; a fact which we summarize as necessary condition 2 below.

Necessary Condition 2:If $\beta$ is a canonical blocking vertex assignment for a $q$-persistent graph determined by a realization $P$, then $\beta$ is path-symmetric.

The two remaining necessary conditions reflect the constraints imposed on occluding paths, generated by canonical blocking vertex assignments, because of their correspondence with Euclidean shortest paths A blocking vertex assignment satisfying these two conditions will be called a path-consistent assignment.

Necessary Condition 3: If $\beta$ is a canonical blocking vertex assignment for a q-persistent graph determined by a realzzation $P$, and if $u_{x} \in \operatorname{path}_{\beta}\left(v_{2}, u_{y}\right)$, and $u_{y} \in \operatorname{path}_{\beta}\left(u_{x}, v_{k}\right)$ then $u_{x}, u_{y} \in \operatorname{path}_{\beta}\left(v_{\imath}, v_{k}\right)$.

Necessary Condition 4: If $\beta$ is a canonical blocking vertex assignment for a $q$-persistent graph determined by a realization $P$, and

1. If $v_{p} \in \operatorname{path}_{\beta}\left(v_{i}, v_{k}\right)$ is an inner blocking vertex for $v_{z} v_{k}$, then for all $v_{x}$ on $\operatorname{chain}\left[v_{i}, v_{p}\right)$ and $v_{y}$ on $\operatorname{chain}\left(v_{p}, v_{k}\right], v_{p} \in \operatorname{path}_{\beta}\left(v_{x}, v_{y}\right)$

2. If $v_{p} \in \operatorname{path}_{\beta}\left(v_{i}, v_{k}\right)$ is an outer blocking vertex for $v_{i} v_{k}$, then for all $v_{x}$ on $\operatorname{chain}\left(v_{p}, v_{i}\right]$ and $v_{y}$ on $\operatorname{chain}\left[v_{k}, v_{p}\right), v_{p} \in \operatorname{path}_{\beta}\left(v_{x}, v_{y}\right)$

The proofs of necessary conditions 3 and 4 are based on the fact that Euclidean shortest paths satisfy the above combinatorial conditions. It is natural to ask whether all the above four conditions are independent of each other. It can be shown that in fact they are. Namely, for any subset of these conditions, there exist q-persistent graphs for which blocking vertex assignments can be constructed that satisfy only that subset and no the others. On the other hand to contrast these conditions with Ghosh's conditions, Everett has exhibited a graph that satisfies Ghosh's conditions and yet it is not a visibility graph. It can be shown that Everett's example is a q-persistent graph, that does not have a blocking vertex assignment that satisfies condition 1 . The graph in figure 1 is a q-persistent graph that was shown not to be a visibility graph in [2]. It can be shown that this graph has one blocking vertex assignment that satisfies necessary condition 1, and another that satisfies conditions 2, 3 and 4, but no one that satisfies all conditions simultaneously. Thus these four necessary conditions are a non-trivial strengthening of Ghosh's conditions. The key question is whether they are sufficient. The next section provides a partial answer to this question. 


\section{Q-Persistent Graphs and Oriented Matroids}

We consider the problem of determining, given as input a q-persistent graph with a blocking vertex assignment satisfying the conditions of the previous section, a combinatorial representation of a potential polygon whose visibility graph is isomorphic to the given graph. The main result of this section is that such a combinatorial reconstruction seems to be significantly easier than the actual reconstruction of the polygon.

Oriented matroids are a well studied combinatorial representation $[6,10$, 15] for point configurations. In the following, we adopt the conventions of [6] and identify oriented matroids with their representations by chirotopes. An equivalence proof for this representation and the classical definition in terms of signed circuits of matroids may be found in [15]. We are concerned here, only with the definition of oriented matroids of rank 3 . Let $\mathcal{T}_{n}, \quad n \geq 3$ denote the set of increasing triples from the set $\{0, \ldots, n-1\}$ ( i.e 3 -tuples $(i, j, k)$ where $i<j<k$ ). A mapping $\chi: \mathcal{T}_{n} \rightarrow\{-1,+1,0\}$ (alternatingly extended to the set of all ordered triples from $\{0, \ldots, n-1\})$ is called a chirotope if for all $i, 0 \leq i \leq n-1$ and all 4-tuples $0 \leq j<k<l<m \leq n-1$ from $\{0, \ldots n-1\}$ the set

$$
\left\{\begin{array}{c}
\chi(i, j, k) \cdot \chi(i, l, m), \\
-\chi(i, j, l) \cdot \chi(i, k, m), \\
\chi(i, j, m) \cdot \chi(i, k, l)
\end{array}\right\}
$$

either contains $\{-1,+1\}$ or equals $\{0\}$. The chirotope is called simplicial if its image is contained in the set $\{-1,+1\}$.

A chirotope is called co-ordinatizable if there exists an $n \times 3$ matrix $M$ such that for any triple $(i, j, k) \in \mathcal{T}_{n}, \chi(i, j, k)$ agrees with the sign of the corresponding $3 \times 3$ subdeterminant of $M$. The chirotope associated with a point configuration assigns to each triple of points its orientation ( given by the signed area). The fact that these subdeterminants obey the chirotope conditions above follows from the well known Grassman-Plucker identities (see [6]). Deciding if a given rank 3 oriented matroid is co-ordinatizable is known to be NP-hard [20]. It is also polynomially equivalent to the decision problem for the existential theory of the reals [17] and thus in PSPACE [7].

We now establish the existence of a simplicial chirotope, corresponding to every q-persistent graph $G$ with a blocking vertex assignment $\beta$ that is pathsymmetric, path consistent and locally-separable. Call such $\beta$ a feasible blocking vertex assignment. The chirotope has the property that any of its coordinatizations defines a simple polygon whose visibility graph is isomorphic to the input graph and induces a canonical blocking vertex assignment on $G$ which is exactly $\beta$. This chirotope, called the Normal Chirotope for the pair $(G, \beta)$ can be constructed in polynomial time given $G$ and $\beta$.

Given a q-persistent graph $G$ with a feasible blocking vertex assignment $\beta$, we define a function $\chi_{G, \beta}: \mathcal{T}_{n} \rightarrow\{-1,+1\}$ (alternatingly extended to the set of 
all ordered triples in $\{0, \ldots, n-1\})$ where

$$
\chi(i, j, k)=\left\{\begin{aligned}
-1 & \text { if there exists } \\
& \text { an occluding path } \\
& \text { generated by } \beta \text { that } \\
& \text { contains the vertices } \\
& v_{i}, v_{j} \text { and } v_{k} \\
+1 & \text { otherwise. }
\end{aligned}\right.
$$

The function $\chi$ can be constructed from $G$ and $\beta$ in $O\left(n^{4}\right)$ time. Moreover, it defines a simplicial chirotope. This constitutes the main result of this paper.

Theorem 4. If $G$ is a q-persistent graph and $\beta$ is a blocking vertex assignment that is path-symmetric, path-consistent and locally-separable then $\chi G, \beta$ is a simplacial chirotope.

We now sketch a proof of the fact that if the normal chirotope $\chi_{G, \beta}$ is realizable, then the corresponding realization yields a polygon whose visibility graph is isomorphic to $G$ and such that the canonical assignment induced on the graph by the polygon is precisely $\beta$. Suppose that a normal chirotope is affinely co-ordinatizable. We first note that points $v_{0}^{*}, \ldots, v_{n-1}^{*}$ in a plane realization of the chirotope, together with the segments $v_{i}^{*} v_{i+1}^{*} \bmod n$ constitute a simple polygon $P$. To see this notice that if $\beta$ is feasible, it is impossible that $\chi(i, i+$ $1, j) \cdot \chi(i, i+1, j+1)=-1$ and $\chi(j, j+1, i) \cdot \chi(j, j+1, i+1)=-1$ when $|i-j|>1$. In the realization this implies that no two segments of the polygon intersect, ensuring simplicity. Also note that since the chirotope is simplicial, the resulting point configuration is always non-degenerate.

Now consider a triple $v_{z} v_{p} v_{q}$ in $G$ such that $v_{\imath}$ is adjacent to no vertex in chain $\left(v_{p}, v_{q}\right)$ and adjacent to $v_{p}$ and $v_{q}$. Let $v_{k} v_{k+1}$ be the split edge for the triple determined by $\beta$. Interpreting signs assigned to the ordered triples by $\beta$ as orientations of the corresponding triples of points, we can show that

1. The interior of the triangle $v_{i}^{*} v_{p}^{*} v_{q}^{*}$ contains no points of $P$.

2. The points corresponding to chain $\left(v_{p}, v_{k}\right]$ and the points corresponding to chain $\left[v_{k+1}, v_{q}\right]$ lie on opposite halfspaces of the line containing $v_{\imath}^{*} v_{p}^{*}$. Similarly the points corresponding to chain $\left[v_{p}, v_{k}\right]$ and the points corresponding to chain $\left[v_{k+1}, v_{q}\right)$ lie on opposite halfspaces of the line containing $v_{\imath}^{*} v_{q}^{*}$.

We also note that by local-inseparability $v_{\imath}$ cannot lie both on an occluding path from $v_{p}$ to a vertex on $\operatorname{chain}\left(v_{i}, v_{p}\right)$ and also on a path from $v_{q}$ to one on chain $\left(v_{q}, v_{i}\right)$. This together with item 1 allow us to claim that $v_{i}^{*}, v_{p}^{*}$, and $v_{q}^{*}$ are visible from each other. Item 2 allows us to claim that $v_{i}^{*}$ is invisible from all the points corresponding to those on $\operatorname{chain}\left(v_{p}, v_{q}\right)$.

Therefore $v_{p}^{*}$ and $v_{q}^{*}$ are succesive neighbors of $v_{\imath}^{*}$ and $v_{k}^{*} v_{k+1}^{*}$ is the split segment for this triple. A similar argument shows the converse case, that is when $v_{p}^{*}$ and $v_{q}^{*}$ are the succesive neighbors of a vertex $v_{\imath}^{*}$, then the corresponding three vertices are all adjacent to each other. Also, if the split segment determined 
by the realization is $v_{k}^{*} v_{k+1}^{*}$, then the corresponding split edge determined by $\beta$ for this triple is $v_{k} v_{k+1}$. Repeating the argument for each triple in $G$ shows that the co- ordinatization gives a simple polygon $P$ whose visibility graph is $G$, and determines the canonical assignment $\beta$ on $G$.

From the previous discussion it follows that any coordinatization of the chirotopes described here will turn theorem 4 into the first characterization of visibility graphs. We know at this point such a characterization holds for values of $\mathrm{n}$ up to 7 and for visibility graphs of 2-spiral polygons. We notice in closing that all the conditions stated in the hypothesis of theorem 4 are used in its proof.

\section{References}

1. J. Abello, O.Egecioglu, Visibality Graphs of Staircase Polygons with Unaform Step Length, International Journal of Discrete and Computational Geometry, 3 (1993) pp. 27-37.

2. J. Abello, L. Hua, C. Pisupati, On Visibility Graphs of Simple Polygons, Congressus Numerantium, 90 (1992), pp. 119-128.

3. J.Abello, O.Egecioglu, K.Kumar, Vusibulity Graphs of Staircase Polygons and the Weak Bruhat Order I : From Visibility Graphs to Maximal Chains, Discrete and Computational Geometry, Accepted (pending revisions).

4. J. Abello, K. Kumar, Visibility Graphs and Oriented Matroids (Long Version), Manuscript.

5. B. Aronov, On the Geodesic Voronoi Diagram of Point Sites in a Simple Polygon, Algorithmica, 4 (1989) pp. 109-140.

6. J. Bokowski, B. Sturmfels, Computational Synthetıc Geometry, Lecture Notes in Mathematics, 1335 (1989), Springer- Verlag, New York.

7. J. Canny, Some Algebraic and Geometric Computations in PSPACE, Proceedings of the 20th ACM Symposium on Theory of Computing, 1988, pp. 460-467.

8. C. Coullard and A. Lubiw, Distance Visibality Graphs, Proceedings of the ACM Symposium on Computational Geometry, 1991, pp. 290-302.

9. H. Everett, Visıbilaty Graph Recognation, PhD Dissertation, Department of Computer Science, University of Toronto, 1990.

10. J. Folkman, J. Lawrence, Oriented Matrouds, Journal of Combinatorial Theory (B), 25 (1978), pp. 199-236.

11. S.K. Ghosh, On Recognızıng and Characterizıng Visibility Graphs of Simple Polygons, Lecture Notes in Computer Science, 318 (1988), pp. 132- 139, SpringerVerlag, New York.

12. J.E Goodman, R. Pollack, "Semispaces of Configurations, Cell Complexes of Arrangements," Journal of Combinatorial Theory (A), (37) pp. 84-102, 1984.

13. N. Greene, M. Kass, and G. Miller, Hzerarchical Z-buffer Visıbılıty, SIGGRAPH 93 Proceedings, pp 231-238, 1993.

14. K. Kumar, Combinatoral Aspects of Point Visibility, PhD. Dissertation, Dept.. of Computer Science, Texas A\&M University, August 1993.

15. J. Lawrence, Oriented Matroids and Multiply Ordered Sets, Linear Algebra and its Applications, 48 (1982) pp. 1-12. 
16. T. Lozano-Perez and M. A. Wesley "An Algorithm for Planning Collision-Free Paths among Polyhedral Obstacles," Communications of the ACM, (22) pp. 560$570,1979$.

17. N.E. Mnëv, The Universality Theorems on the Classification Problem of Cionfiguration Varzeties and Convex Polytope Varietzes, Lecture Notes in Mathematics, 1346 (1988), pp. 527-544, Springer-Verlag, New York.

18. J. O'Rourke, Art Gallery Theorems and Algorithms, Oxford University Press, 1987.

19. J. O'Rourke, The Computational Geometry Column, SIGACT News, (1992).

20. P.W. Shor, Stretchability of Pseudolines is NP-Hard, DIMACS Series in Discrete Mathematics and Theoretical Computer Science, 4 (1991), pp. 531-554.

21. S. Teller, P. Hanrahan, Global Visibility Algorithms for Ilumination Computations, SIGGRAPH 93 Proceedings, pp 239-246, 1993. 